ARTICLE

Received 21 Dec 2012 | Accepted 26 Apr 2013 | Published 4 Jun $2013 \quad$ DOl: 10.1038/ncomms2941

\title{
Stable Li-ion battery anodes by in-situ polymerization of conducting hydrogel to conformally coat silicon nanoparticles
}

Hui Wu ${ }^{1,2, \star}$, Guihua Yu ${ }^{3, \star}$, Lijia Pan ${ }^{4,5}$, Nian Liu' ${ }^{1}$, Matthew T. McDowell ${ }^{1}$, Zhenan Bao ${ }^{4}$ \& Yi Cui ${ }^{1,6}$

\begin{abstract}
Silicon has a high-specific capacity as an anode material for Li-ion batteries, and much research has been focused on overcoming the poor cycling stability issue associated with its large volume changes during charging and discharging processes, mostly through nanostructured material design. Here we report incorporation of a conducting polymer hydrogel into Si-based anodes: the hydrogel is polymerized in-situ, resulting in a well-connected threedimensional network structure consisting of $\mathrm{Si}$ nanoparticles conformally coated by the conducting polymer. Such a hierarchical hydrogel framework combines multiple advantageous features, including a continuous electrically conductive polyaniline network, binding with the Si surface through either the crosslinker hydrogen bonding with phytic acid or electrostatic interaction with the positively charged polymer, and porous space for volume expansion of $\mathrm{Si}$ particles. With this anode, we demonstrate a cycle life of 5,000 cycles with over $90 \%$ capacity retention at current density of $6.0 \mathrm{Ag}^{-1}$.
\end{abstract}

\footnotetext{
${ }^{1}$ Department of Materials Science and Engineering, Stanford University, Stanford, California 94305, USA. ${ }^{2}$ State Key Laboratory of New Ceramics and Fine Processing, School of Materials Science and Engineering, Tsinghua University, Beijing 100084, China. ${ }^{3}$ Materials Science and Engineering Program and Department of Mechanical Engineering, The University of Texas at Austin, Austin, Texas 78712, USA. ${ }^{4}$ Department of Chemical Engineering, Stanford University, Stanford, California 94305, USA. ${ }^{5}$ National Laboratory of Microstructures (Nanjing), School of Electronic Science and Engineering, Nanjing University, Nanjing 210093, China. ${ }^{6}$ Stanford Institute for Materials and Energy Sciences, SLAC National Accelerator Laboratory, 2575 Sand Hill Road, Menlo Park, Stanford, California 94025, USA. * These authors contributed equally to this work. Correspondence and requests for materials should be addressed to Z.B. (email: zbao@stanford.edu) or to Y.C. (email: yicui@stanford.edu).
} 
D eveloping rechargeable lithium-ion batteries with highenergy density and long cycle life is of critical importance to address the ever-increasing energy storage needs for various technological applications, including portable electronics, hybrid and electric vehicles, and grid-scale energy storage systems ${ }^{1-4}$. Graphite, the traditional anode material in lithiumion batteries does not meet with high energy needs due to its limited theoretical specific capacity of $\sim 370 \mathrm{mAhg}^{-1}$. Silicon (Si) has attracted a great deal of attention as an alternative anode material for Li-ion batteries lately ${ }^{5-8}$, primarily due to: (1) its high theoretical gravimetric capacity of $\sim 4,200 \mathrm{mAh} \mathrm{g}^{-1}$ based on the Si weight, (2) its relatively low discharge potential $(\sim 0.5 \mathrm{~V}$ versus $\left.\mathrm{Li} / \mathrm{Li}^{+}\right)$, (3) the natural abundance of elemental $\mathrm{Si}$, and (4) its safety and environmental benignity ${ }^{9-17}$.

However, Si-based anodes also face significant challenges due to the $\sim 400 \%$ volume expansion upon lithium insertion in $\mathrm{Si}^{18}$; this can result in fracture and loss of electrical contact, and an unstable solid electrolyte interphase (SEI) growth on the Si surface $^{19}$. Recently, exciting progress has been made through nanostructuring of Si. Nanocrystals ${ }^{20}$, nanowires ${ }^{21}$, core-shell nanofibers ${ }^{22,23}$, nanotubes ${ }^{16}$, nanospheres ${ }^{24}$, nanoporous materials $^{25}$ and $\mathrm{Si} /$ carbon nanocomposites ${ }^{26,27}$ have all shown longer cycling life as candidate anode materials compared with micron-sized $\mathrm{Si}$ particles ${ }^{12,13,16,21,22,25-27}$. In addition, fundamental studies using in-situ X-ray, electron microscopy, and mechanical modelling have helped elucidate the nature of $\mathrm{Li}-\mathrm{Si}$ reactions ${ }^{6-8,28,29}$. However, synthesis of $\mathrm{Si}$ nanostructures usually involves high temperature chemical vapour deposition or complex chemical reactions and/or templates, ${ }^{11,21-25}$ and their scalability and compatibility with existing battery manufacturing processes remain a challenge.

More recently, electrodes made from Si nanoparticle (SiNP) slurries have been investigated by several research groups as a potentially manufacturing-compatible route $e^{25,26,30,31}$. Various polymer binders other than traditional polyvinylidene difluoride (PVDF), including polyacrylic acid (PAA), carboxy-methyl cellulose (CMC), poly(9,9-dioctylfluorene-co-Suorenone-comethylbenzoic acid) (PFFOMB) and alginate, have been found to enhance the cycle life ${ }^{14,15,32-36}$. The cycle life improvement when using PAA, CMC and alginate binders was attributed to the high polymer modulus values and the binding between the functional groups on the polymers with the surface oxide on the Si particles. In the case of the PFFOMB binder, an interesting concept was demonstrated that combined binding and conducting properties in the binder molecules ${ }^{5}$.

Building on the previous work on polymer binders, here we demonstrate that a unique feature of our approach for realizing this excellent performance is the use of in-situ polymerization, to form a bi-functional conformal coating that binds to the $\mathrm{Si}$ surface and also serves as a continuous three-dimensional (3D) pathway for electronic conduction. The in-situ polymerization fabrication technique is quite different from previous studies, where the direct mixing of $\mathrm{Si}$ particles with polymer binders produces less conformal coatings and non-continuous electronic transport pathway.

\section{Results}

Material and structural design. Conductive polymer hydrogels are unique materials that offer advantages such as a $3 \mathrm{D}$ hierarchical porous-conducting framework and excellent electronic and electrochemical properties ${ }^{37}$. Recently, we have shown that they exhibit superior electrochemical performance when used in supercapacitors and ultrasensitive biosensors ${ }^{38}$. The $3 \mathrm{D}$ porous micro- and nanostructures of conductive polymer hydrogels can promote the transport of both electrons and ions owing to the availability of short diffusion paths and good $3 \mathrm{D}$ connectivity. Moreover, they can be synthesized by simply mixing two solutions, where one contains the initiator (oxidizer) and the other contains the crosslinker and the monomer. For example, phytic acid, a naturally occurring molecule consisting of six phosphoric acid groups, can be used as both the gelator and dopant to react with the aniline monomer by protonating the nitrogen groups on polyaniline (PANi), leading to the formation of a $3 \mathrm{D}$ interconnected network structure ${ }^{38}$. In this work, when SiNPs are mixed into the solution during polymerization, the phosphoric acid groups in the phytic acid molecules can potentially bind with the $\mathrm{SiO}_{2}$ on the $\mathrm{Si}$ particle surfaces via hydrogen bonding, which was thought to contribute to the improved cycle lifetime for PAA, CMC and alginate binders with carboxylic acid groups ${ }^{32-34,36}$. This interaction can also result in the conformal coating of phytic acid molecules on the surface, which may further crosslink with aniline monomers during polymerization to generate a conformal conductive coating. Additionally, the negatively charged surface oxide may electrostatically interact with the positively charged PANi doped by the phytic acid ${ }^{39}$. All the above interactions result in the formation a 3D continuous-conducting hydrogel network, trapping the coated Si particles inside (Fig. 1a).

Material synthesis and electrode fabrication. The SiNP-PANi hydrogel composite is prepared via a scalable solution phase synthesis by mixing SiNPs with phytic acid and aniline in water to give a brown suspension (Fig. 1b). Within 3 min upon adding an oxidizer (for example, ammonium persulphate), the aniline was observed to rapidly polymerize and crosslink, resulting in a dark green viscous gel due to the presence of the phytic acid gelator (Fig. 1c). The viscous gel was then bladed onto a copper foil current collector and dried to form a uniform film over a large area; Fig. 1d shows an example of a uniformly coated electrode film $(5 \mathrm{~cm} \times 20 \mathrm{~cm})$. This solution-based synthesis method and its compatibility with roll-to-roll coating methods make this system readily scaled for large area electrode films ${ }^{40}$. The SiNP-PANi hydrogel composite film was then thoroughly washed in deionized water to remove excess ions and oligomers, followed by vacuum drying overnight.

Figure 2 shows scanning electron microscopy (SEM) images of the SiNP-PANi hydrogel composite electrodes. Typically, the spherical silicon nanoparticles have an average diameter of $\sim 60 \mathrm{~nm}$ (Fig. 2a), while the dried polymer hydrogel consists of a hierarchical 3D porous foam-like network composed of dendritic nanofibers with typical diameters of $60-100 \mathrm{~nm}$ (Fig. 2b). As the PANi was formed in the presence of the SiNPs, the SiNPs are in intimate contact with the conductive polymer hydrogel matrix at both the microscopic and molecular level. This is confirmed by SEM images of the composite electrode (Fig. 2c), which clearly show a uniform mixture of SiNPs embedded inside the highly porous polymer matrix. Additionally, SiNPs appear to be encapsulated by a conformal PANi coating due to the in-situ polymerization discussed above, as shown in the transmission electron microscopy (TEM) image (Fig. 2d). As will be discussed later, the superior electrochemical performance of the Si-PANi hydrogel composite electrodes can be attributed to the advantageous features offered by the unique hierarchical microstructure.

Electrochemical performance. To characterize the electrochemical properties of SiNP-PANi composite electrodes, cyclic voltammetry $(\mathrm{CV})$ measurements were performed on half cells at a scan rate of $0.1 \mathrm{mV} \mathrm{s}^{-1}$ over the potential window of $0.01-1 \mathrm{~V}$ versus $\mathrm{Li} / \mathrm{Li}^{+}$(Fig. 3a). As shown in Fig. 3a, the CV profile (red curve) of a SiNP-PANi hydrogel composite electrode exhibits 


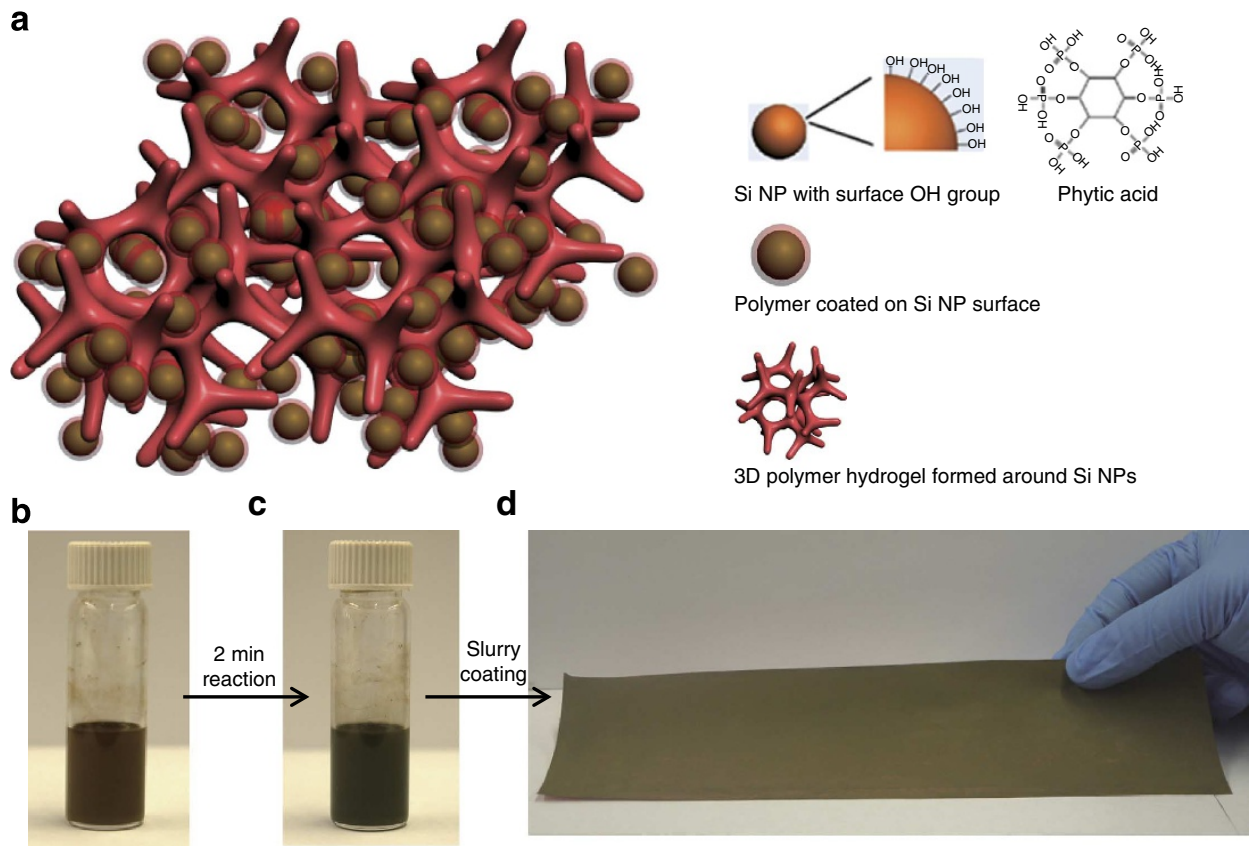

Figure 1 | Electrode design and fabrication. (a) Schematic illustration of 3D porous SiNP/conductive polymer hydrogel composite electrodes. Each SiNP is encapsulated within a conductive polymer surface coating and is further connected to the highly porous hydrogel framework. SiNPs has been conformally coated with a polymer layer either through interactions between surface - $\mathrm{OH}$ groups and the phosphonic acids in the crosslinker phytic acid molecules (right column), or the electrostatic interaction between negatively charged -OH groups and positively charge PANi due to phytic acid doping. (b-d) Photographs showing the key steps of the electrode fabrication process. (b) First, SiNPs were dispersed in the hydrogel precursor solution containing the crosslinker (phytic acid), the monomer aniline and the initiator ammonium persulphate. (c) After several minutes of chemical reaction, the aniline monomer was crosslinked, forming a viscous gel with a dark green colour. (d) The viscous gel was then bladed onto a $5 \times 20 \mathrm{~cm}^{2}$ copper foil current collector and dried.

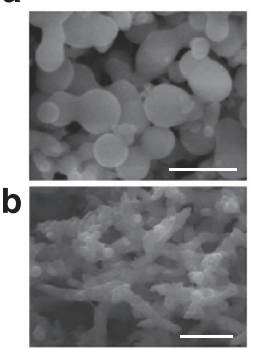

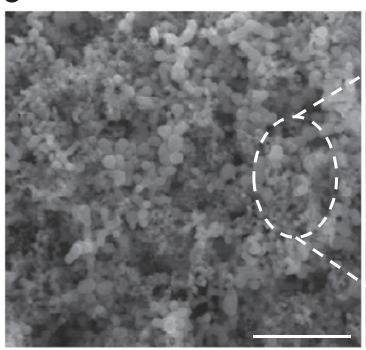

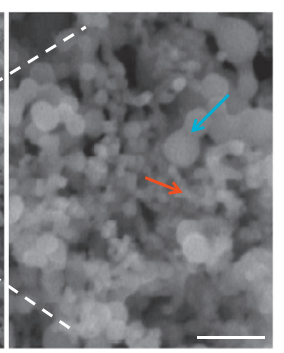

d

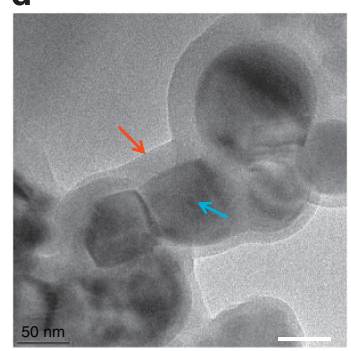

Figure 2 | Microstructure of the SiNP-PANi composite electrodes. (a) SEM image of pure SiNPs. Scale bar, 200 nm. (b) SEM image of the PANi hydrogel sample. Scale bar, $100 \mathrm{~nm}$. (c) SEM image of a SiNP-PANi composite electrode at low (left) and high (right) magnifications. Scale bar, $1 \mu \mathrm{m}$ (left) and $200 \mathrm{~nm}$ (right). The blue arrow indicates SiNPs while the red arrow shows the PANi hydrogel network. (d) A TEM image shows that the SiNPs (blue arrow) are coated with a uniform PANi polymer layer (red arrow). Scale bar, $50 \mathrm{~nm}$.

similar electrochemical characteristics as that of pristine $\mathrm{Si}$ powders ${ }^{26}$. The peak at $0.19 \mathrm{~V}$ in the cathodic process corresponds to the conversion of crystalline $\mathrm{Si}$ to the $\mathrm{Li}_{\mathrm{x}} \mathrm{Si}$ phase, while the two peaks at 0.41 and $0.53 \mathrm{~V}$ in the anodic process correspond to the delithiation of amorphous $(\alpha) \alpha-\mathrm{Li}_{\mathrm{x}} \mathrm{Si}$ to $\alpha-\mathrm{Si}^{26}$. For comparison, the CV profile of a dried PANi hydrogel film (blue line in Fig. 3a) shows that the current density is about two orders of magnitude lower than that of the composite electrode, indicating negligible contribution from PANi to the capacity of the whole electrode.

The electrochemical cycling performance of the composite electrodes was evaluated using deep charge/discharge galvanostatic cycling from 1 to $0.01 \mathrm{~V}$ (Fig. 3b-e). The first charge has a long plateau around $0.1 \mathrm{~V}$, which corresponds to the lithiation potential of pure crystalline silicon (Supplementary Fig. S1). At a charge/discharge current of $1.0 \mathrm{Ag}^{-1}$, the SiNP-PANi composite electrode exhibits a relatively stable reversible lithium capacity of $1,600 \mathrm{mAh} \mathrm{g}^{-1}$ for 1,000 deep cycles based on the weight of only $\mathrm{Si}$ (Fig. 3b). In comparison, the SiNP electrode using traditional PVDF binder loses $>50 \%$ of its initial capacity after being cycled only 100 times (Fig. 3b). The volumetric capacity of the SiNPPANi hybrid electrode is estimated to be $\sim 1,080 \mathrm{mAh} \mathrm{cm}^{-3}$ (see supporting information for calculation details), which is about double that of graphite electrode $\left(\sim 620 \mathrm{mAh} \mathrm{cm}^{-3}\right)^{33}$, but lower than the volumetric capacity for SiNP-alginate electrode reported in Kovalenko et al. ${ }^{33}\left(\sim 1,520 \mathrm{mAh} \mathrm{cm}^{-3}\right)$. This is 
a

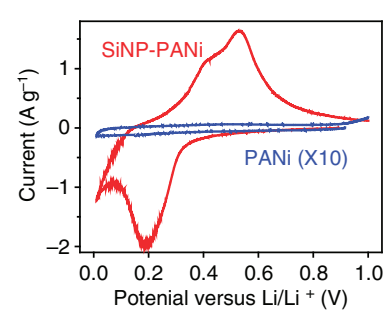

c
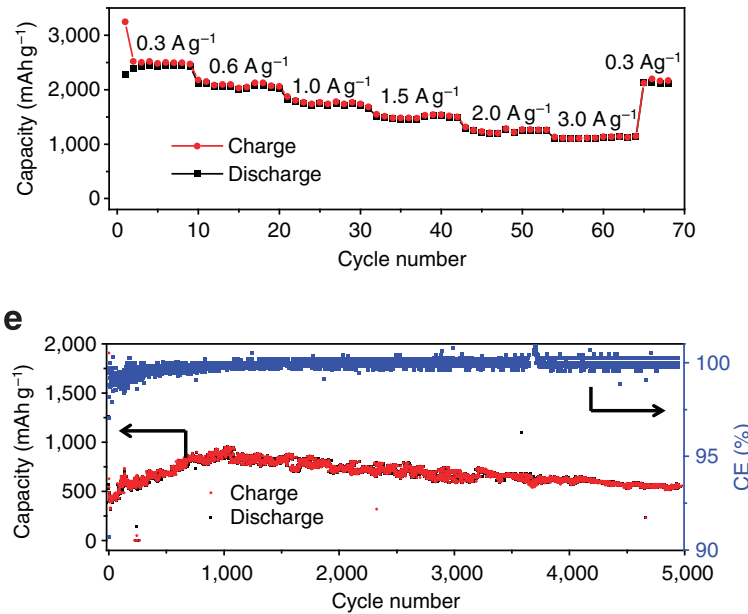

b

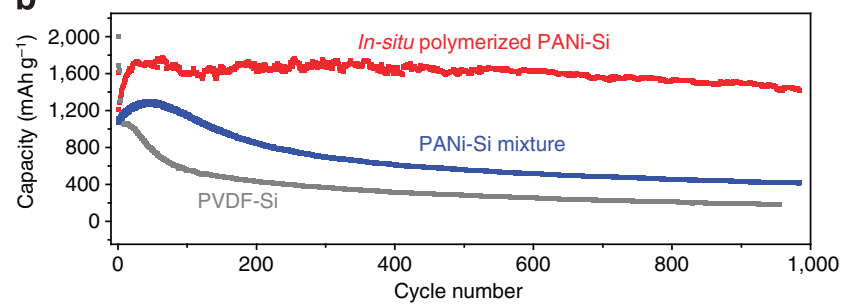

d

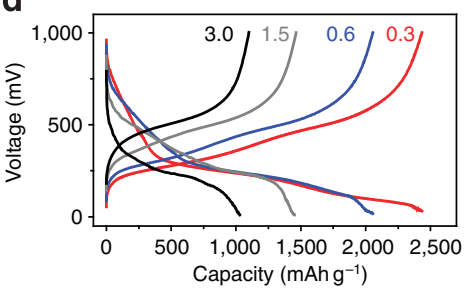

f

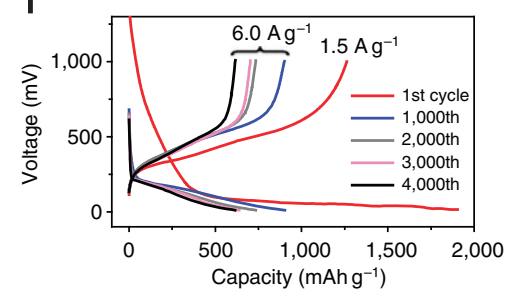

Figure 3 | Electrochemical characteristics. (a) CV measurement of SiNP-PANi hydrogel composite (red line) and PANi hydrogel (blue line) at a scan rate of $0.1 \mathrm{mVs}^{-1}$ over the potential window of $0.01-1 \mathrm{~V}$ versus $\mathrm{Li} / \mathrm{Li}+$. (b) Electrochemical cycling performance of the in-situ polymerized SiNP-PANi composite electrodes under deep charge/discharge cycles from 1 to $0.01 \mathrm{~V}$ with a charge/discharge current of $1.0 \mathrm{Ag}^{-1}$, showing stable capacity of $\sim 1,200 \mathrm{mAh}^{-1}$ after 1,000 cycles (red line). Electrochemical cycling of control samples are compared in the figure. Blue line: SiNP-PANi without in-situ polymerization. Green line: PVDF-SiNP electrode. (c) Capacity and (d) galvanostatic charge/discharge profiles of a SiNP-PANi electrode cycled at various current densities: $0.3,0.6,1.0$ and $3.0 \mathrm{Ag}^{-1}$ (from right to left). (e) Lithiation/delithiation capacity and CE of SiNP-PANi electrode cycled at current density of $6.0 \mathrm{Ag} \mathrm{g}^{-1}$ for 5,000 cycles. (f) Galvanostatic charge/discharge profiles plotted for the 1st, 1,000th, 2,000th, 3,000th and 4,000th cycles. All electrochemical cycling measurements (b-f) were carried out at room temperature in two-electrode 2032 coin-type half-cells. All the specific capacities and current density are reported based on the weight of SiNPs.

mainly due to the $3 \mathrm{D}$ porous structure of the whole electrode as presented in Fig. 2c. The active Si material mass loading has an important role for practical battery applications. The Si mass loading in the present study is typically $0.2-0.3 \mathrm{mg} \mathrm{cm}^{-2}$, which is similar to that of $\mathrm{Si} / \mathrm{PFFOMB}$ electrode reported by Liu et al. ${ }^{32}$ $\left(0.3 \mathrm{mg} \mathrm{cm}^{-2}\right.$ for total mass loading, and $0.2 \mathrm{mg} \mathrm{cm}^{-2} \mathrm{Si}$ mass loading). We note that although this value is low for practical battery application, the mass loading can be potentially increased by multiple depositions of materials, which is an important aspect to investigate for future studies.

The continuous-conducting polymer hydrogel framework, which is directly connected to the current collector, provides channels for fast electron transport, thereby enabling outstanding rate capability. As shown in Fig. 3c, the capacity of a SiNP-PANi composite electrode varies from $2,500 \mathrm{mAhg}^{-1}$ to $1,100 \mathrm{mAhg}^{-1}$ at charge/discharge rate from 0.3 to $3.0 \mathrm{Ag}^{-1}$. Fig. 3d shows that even at a high charge/discharge current of $3.0 \mathrm{Ag}^{-1}$, the lithiation potential still shows a sloping profile between 0.3 and $0.01 \mathrm{~V}$, consistent with previously reported $\mathrm{Li}$ insertion to form amorphous $\mathrm{Li}_{\mathrm{x}} \mathrm{Si}^{11}$. Clearly, $\mathrm{Li}^{+}$ions can rapidly pass through the thin PANi layer to reach the silicon active material even at high charge/discharge rates.

To obtain long cycling results within a reasonable testing period, faster charge and discharge tests with deep cycling (1 to $0.01 \mathrm{~V}$ ) were carried out. Figure $3 \mathrm{e}$ shows that at a high current density of $6.0 \mathrm{Ag}^{-1}$, an electrode capacity of $\sim 550 \mathrm{mAhg}^{-1}$ was still retained after 5,000 cycles, which corresponds to $\sim 91 \%$ capacity retention at current density of $6.0 \mathrm{Ag}^{-1}$. No obvious changes in the charge capacity or charge/discharge profiles can be found after 5,000 cycles for the Si-PANi hybrid anode (Fig. 3f), indicating its superior and stable cycling performance. We note that an increase of the capacity of the in-situ polymerized PAN-Si anode was observed during the initial cycles (Fig. 3b,e). The origin of this activation step can be possibly attributed to the delayed wetting of electrolyte into the $3 \mathrm{D}$ nanoporous structure of the composite electrode. High coulombic efficiency (CE) is required for practical silicon-based electrodes. For our Si-PANi hydrogel composite electrodes, the $\mathrm{CE}$ of the first cycle was usually $\sim 70 \%$ because SEI formation consumes a certain percentage of the lithium; this could be improved by prelithiation in future studies (Fig. 3e,f). The average CE of the composite electrode from the 2 nd to 5,000th cycle is $99.8 \%$, which is higher than that previously reported for silicon nanoparticles and nanowires $^{21,25}$. The high $\mathrm{CE}$ achieved here is due in large part to the formation of a stable SEI on the composite electrode, which we will discuss in greater details later.

\section{Discussion}

We attribute the above demonstrated exceptional electrochemical stability to the unique nanoscale architecture of the Si-PANi composite electrode. First, the porous hydrogel matrix has empty space to allow for the large volume expansion of the SiNPs during lithium insertion. Second, the highly conductive and continuous 
a

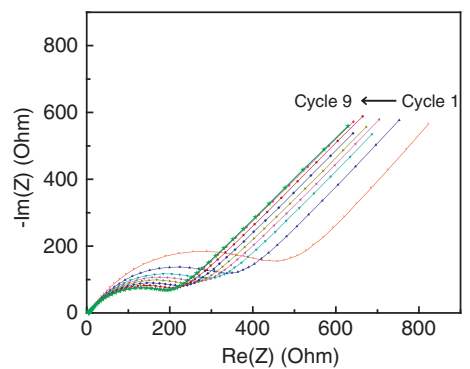

b

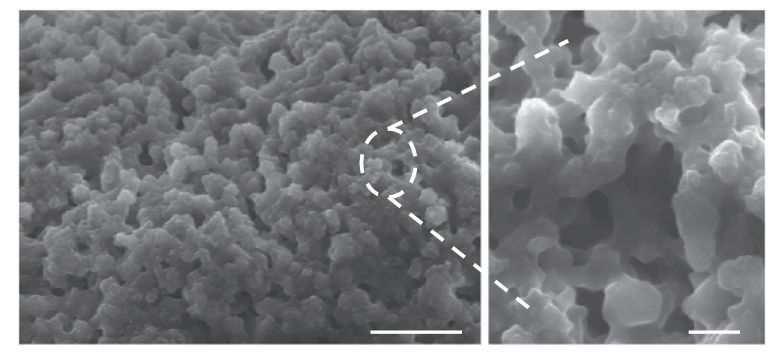

Figure 4 | Cell impedance test and characterization of SEl formation on composite electrodes. (a) Cell impedance tests of the SiNP-PANi composite electrode after each cycle between cycles 1 and 9. (b) SEM images of the electrode after 2,000 electrochemical cycles, showing that a uniform and thin SEI forms on the composite electrode. Scale bar, $1 \mu \mathrm{m}$ (left) and $200 \mathrm{~nm}$ (right).

3D PANi framework, as well as the conformal conductive coating surrounding each SiNP, helps provide good electrical connection to the particles. Third, although pulverization of larger particles may still occur during lithiation and battery cycling, the fractured $\mathrm{Si}$ pieces are trapped within the interconnected narrow pores of the polymer matrix, maintaining good electrical connectivity among fractured particles. TEM images showed that even after 2,000 electrochemical cycles, SiNPs are still inside the polymer matrix without losing electrical contact (Supplementary Fig. S2). Even though we observed that the polymer surface coating on the particles broke upon initial volume expansion during lithiation, the coating still nonetheless enabled the SiNPs to remain connected to the conductive matrix (Supplementary Fig. S2).

To further confirm the stabilizing effect of the in-situ polymerized PANi coating, we fabricated a control electrode by mixing pre-synthesized PANi hydrogel and SiNPs. In this control sample, a similar weight ratio of the SiNPs to the PANi hydrogel composite structure was used, but there was no intimate surface coating on the Si particles as the aniline precursor had already been polymerized before mixing. The electrochemical cycling of this control sample is presented in Fig. 3b. Even though this system showed better cycling stability than the bare SiNP electrode with PVDF binder, lower capacity retention than the in-situ polymerized PANi-SiNP composite electrode was obtained.

Importantly, the uniform PANi coating on the Si particles also assists in enabling a deformable and stable SEI on the SiNP surface. We performed cell impedance measurements of the composite electrode after different cycles. As shown in Fig. 4a and Supplementary Fig. S3, no obvious impedance increase was observed after cycles, indicating limited growth of the SEI during cycling. The impedance decrease for the first few cycles may be attributed to two major reasons: the slow wetting of electrolyte into porous battery electrodes, and the conductivity increase of SiNPs after lithium-ion doping associated with electrochemical charging. Indeed, as shown in Supplementary Fig. S3, the impedance does not show obvious changes after cycling for more than 200 times. SEM images of composite electrodes after 2,000 electrochemical cycles confirmed that a uniform and thin SEI formed on the electrode, leading to the good cycling performance.

In comparison, very thick SEI layers grow on SiNP electrodes with traditional PVDF binders (Supplementary Fig. S4). The formation of a thin and stable SEI could potentially be attributed to the modification of the Si surface by the in-situ polymerization of PANi, similar to the polar hydrogen bonds between carboxyl groups of some binders and $\mathrm{SiO}_{2}$ reported in recent studies ${ }^{33,41}$. Detailed investigation of this topic will be the subject of future studies. The stable SEI formation on our composite electrodes must be important for the long cycle life, as well as the high CE in the half-cell battery tests.

In conclusion, we have developed a facile and scalable solution process to fabricate high-performance Li-ion battery negative electrodes by encapsulating SiNPs in a nanostructured 3D porous conductive polymer framework. By taking advantage of the conductive polymer matrix, which provides fast electronic and ionic transfer channels, as well as free space for $\mathrm{Si}$ volume changes, we successfully achieved high capacity and extremely stable electrochemical cycling. The electrode can be continuously deep cycled up to 5,000 times without significant capacity decay. Moreover, the solution synthesis and electrode fabrication process are highly scalable and compatible with existing slurry coating battery manufacturing technology. This will potentially allow for this high-performance composite electrode to be scaled up for manufacturing the next generation of high-energy Li-ion batteries, which are important for applications in electric vehicles and grid-scale energy storage systems that require both low-cost and reliable battery systems. In addition, our described materials design for silicon-based anodes may be extended to other battery electrode material systems that experience large volume changes and unstable SEI formation during cycling.

\section{Methods}

Synthesis of electrode materials. Composite SiNP-PANi hydrogel electrodes were made via the following solution processes. First, $0.9 \mathrm{ml}$ solution A, which contains $100 \mathrm{mM}$ aniline monomer and $\sim 30 \mathrm{mM}$ phytic acid, was added and mixed with $80 \mathrm{mg}$ SiNPs (MTI, Inc.). Second, $0.3 \mathrm{ml}$ solution B, containing $125 \mathrm{mM}$ ammonium persulfate, was added into the above mixture and subjected to $\sim 1$ min bath sonication (Supplementary Fig. S5). In about 2 min, the solution changed colour from brown to dark green and became viscous and gel-like, indicating in-situ polymerization of aniline monomer to form the PANi hydrogel.

Electrode fabrication. The SiNP/PANi electrode was made by doctor-blading the viscous SiNP-PANi hydrogel onto a Cu foil current collector (no binder used) and drying at room temperature. The SiNP-PANi hydrogel composite film was then mechanically pressed and thoroughly washed in deionized water several times to remove excess phytic acid, and the composite electrode film was dried in vacuum at room temperature for use in half cells as the working electrode. The total mass loading was typically $0.3-0.4 \mathrm{mg} \mathrm{cm}^{-2}$. The Si mass loading is typically $0.2-0.3 \mathrm{mg} \mathrm{cm}^{-2}$. We measured the yield of PAni in the reaction by obtaining the final weight of PAni hydrogel at the same recipe without the SiNP (after washing excessive monomers, acids and dried). With the yield value, we calculated the percent of $\mathrm{Si}$ in the composite to be $75 \%$, or the Si to conductive hydrogel mass ratio of 3:1. The PANi hydrogel-only control samples were made via the same process by simply mixing the two solutions (solution A and solution B) without the addition of SiNPs.

Cell assembly and electrochemical characterization. The electrochemical properties were examined by galvanostatic cycling of coin-type half cells with the SiNP-PANi composite as the working electrode and lithium foil as the counter/ reference electrode. The electrolyte for all tests was $1 \mathrm{M} \mathrm{LiPF}_{6}$ in ethylene 
carbonate/diethylcarbonate/vinylene carbonate (1:1:0.02 v/v/v, Ferro Corporation), and separators $(25 \mu \mathrm{m})$ from Asahi Kasei Co. were used.

Calculation of volumetric capacity. The thickness of the SiNP-PANi hybrid film was measured to be $\sim 4.45 \mu \mathrm{m}$ for an electrode with mass loading of $0.3 \mathrm{mg} \mathrm{cm}^{-2}$. Therefore, the density $(\rho)$ of the electrode is:

$$
\rho=\frac{0.3 \mathrm{mg} \mathrm{cm}^{-2}}{4.45 \mu \mathrm{m}}=0.674 \mathrm{~g} \mathrm{~cm}^{-3}
$$

The gravimetric capacity $\left(C_{\mathrm{g}}\right)$ of our electrode is measured to be $\sim 1,600 \mathrm{mAh} \mathrm{g}^{-1}$ as reported in Fig. 3 . The volumetric capacity $\left(C_{\mathrm{v}}\right)$ is calculated:

$$
C_{\mathrm{v}}=C_{\mathrm{g}} \times \rho=1,600 \mathrm{mAh} \mathrm{g}^{-1} \times 0.674 \mathrm{~g} \mathrm{~cm}^{-3}=1,078 \mathrm{mAh} \mathrm{cm}^{-3}
$$

\section{References}

1. Armand, M. \& Tarascon, J. M. Building better batteries. Nature 451, 652-657 (2008).

2. Tarascon, J. M. \& Armand, M. Issues and challenges facing rechargeable lithium batteries. Nature 414, 359-367 (2001).

3. Goodenough, I., Abruna, H. \& Buchanan, M. Basic Research Needs for Electrical Energy Storage: Report of the Basic Energy Sciences Workshop on Electrical Energy Storage (US Department of Energy, 2007).

4. Arico, A. S., Bruce, P., Scrosati, B., Tarascon, J. -M. \& van Schalkwijk, W. Nanostructured materials for advanced energy conversion and storage devices. Nat. Mater. 4, 366-377 (2005).

5. Beaulieu, L. Y., Eberman, K. W., Turner, R. L., Krause, L. J. \& Dahn, J. R. Colossal reversible volume changes in lithium alloys. Electrochem. Solid-State Lett. 4, A137-A140 (2001).

6. Hatchard, T. D. \& Dahn, J. R. In situ XRD and electrochemical study of the reaction of lithium with amorphous silicon. J. Electrochem. Soc. 151, A838-A842 (2004).

7. Deshpande, R., Cheng, Y. -T. \& Verbrugge, M. W. Modeling diffusion-induced stress in nanowire electrode structures. J. Power Sources 195, 5081-5088 (2010).

8. Verbrugge, M. W. \& Cheng, Y. -T. Stress and strain-energy distributions within diffusion-controlled insertion-electrode particles subjected to periodic potential excitations. J. Electrochem. Soc. 156, A927-A937 (2009).

9. Besenhard, J. O., Yang, J. \& Winter, M. Will advanced lithium-alloy anodes have a chance in lithium-ion batteries? J. Power Sources 68, 87-90 (1997).

10. Kamali, A. R. \& Fray, D. J. Review on carbon and silicon based materials as anode materials for lithium ion batteries. J. New Mater. Electrochem. Systems 13, 147-160 (2010).

11. Park, C. M., Kim, J. H., Kim, H. \& Sohn, H. J. Li-alloy based anode materials for Li secondary batteries. Chem. Soc. Rev. 39, 3115-3141 (2010).

12. Zhang, W. J. A review of the electrochemical performance of alloy anodes for lithium-ion batteries. J. Power Sources 196, 13-24 (2011).

13. Wu, H. \& Cui, Y. Designing nanostructured Si anodes for high energy lithium ion batteries. Nano. Today 7, 414-429 (2012).

14. Verbrugge, M. W. \& Cheng, Y. T. Stress distribution within spherical particles undergoing electrochemical insertion and extraction. Electrochem. Soc. Trans. 13, 127-139 (2008).

15. Verbrugge, M. W. \& Cheng, Y. T. Stress and strain-energy distributions within diffusion-controlled insertion-electrode particles subjected to periodic potential excitations. J. Electrochem. Soc. 156, A927-A937 (2009).

16. Park, M. H. et al. Silicon nanotube battery anodes. Nano Lett. 9, 3844-3847 (2009).

17. Song, T. et al. Arrays of sealed silicon nanotubes as anodes for lithium ion batteries. Nano. Lett. 10, 1710-1716 (2010).

18. Kasavajjula, U., Wang, C. S. \& Appleby, A. J. Nano- and bulk-silicon-based insertion anodes for lithium-ion secondary cells. J. Power Sources 163, 1003-1039 (2007).

19. Aurbach, D. Review of selected electrode-solution interactions which determine the performance of $\mathrm{Li}$ and $\mathrm{Li}$ ion batteries. J. Power Sources 89, 206-218 (2000).

20. Kim, H., Seo, M., Park, M. H. \& Cho, J. A critical size of silicon nano-anodes for lithium rechargeable batteries. Angew. Chem. Int. Ed. 49, 2146-2149 (2008).

21. Chan, C. K. et al. High-performance lithium battery anodes using silicon nanowires. Nat. Nanotech. 3, 31-35 (2008).

22. Cui, L. F., Ruffo, R., Chan, C. K., Peng, H. L. \& Cui, Y. Crystalline-amorphous core-shell silicon nanowires for high capacity and high current battery electrodes. Nano. Lett. 9, 491-495 (2009).

23. Cui, L. F., Yang, Y., Hsu, C. M. \& Cui, Y. Carbon-silicon core-shell nanowires as high capacity electrode for lithium ion batteries. Nano. Lett. 9, 3370-3374 (2009).
24. Yao, Y. et al. Interconnected silicon hollow nanospheres for lithium-ion battery anodes with long cycle life. Nano. Lett. 11, 2949-2954 (2011).

25. Kim, H., Han, B., Choo, J. \& Cho, J. Three-dimensional porous silicon particles for use in high-performance lithium secondary batteries. Angew. Chem. Int. Ed. 47, 10151-10154 (2008).

26. Magasinski, A. et al. High-performance lithium-ion anodes using a hierarchical bottom-up approach. Nat. Mater. 9, 353-358 (2010).

27. Liu, N. et al. A yolk-shell design for stabilized and scalable li-ion battery alloy anodes. Nano. Lett. 12, 3315-3321 (2012).

28. McDowell, M. T. et al. Studying the kinetics of crystalline silicon nanoparticle lithiation with in situ transmission electron microscopy. Adv. Mater. 24, 6034-6041 (2012).

29. Lee, S. W., McDowell, M. T., Berla, L. A., Nix, W. D. \& Cui, Y. Fracture of crystalline silicon nanopillars during electrochemical lithium insertion. Proc. Natl Acad. Sci. USA 109, 4080-4085 (2012).

30. Hu, L. B. et al. Si nanoparticle-decorated Si nanowire networks for Li-ion battery anodes. Chem. Commun. 47, 367-369 (2011).

31. Wu, H. et al. Engineering empty space between $\mathrm{Si}$ nanoparticles for lithium-ion battery anodes. Nano. Lett. 12, 904-909 (2012).

32. Liu, G. et al. Polymers with tailored electronic structure for high capacity lithium battery electrodes. Adv. Mater. 23, 4679-4683 (2011).

33. Kovalenko, I. et al. A major constituent of brown algae for use in high-capacity li-ion batteries. Science 333, 75-79 (2011).

34. Bridel, J. S., Azaïs, T., Morcrette, M., Tarascon, J. M. \& Larcher, D. Key parameters governing the reversibility of si/carbon/CMC electrodes for li-ion batteries. Chem. Mater. 22, 1229-1241 (2009).

35. Magasinski, A. et al. Toward efficient binders for li-ion battery Si-based anodes: polyacrylic acid. ACS Appl. Mater. Interf. 2, 3004-3010 (2010).

36. Mazouzi, D., Lestriez, B., Roué, L. \& Guyomard, D. Silicon composite electrode with high capacity and long cycle life. Electrochem. Solid-State Lett. 12, A215-A218 (2009).

37. Kopecek, J. Polymer chemistry-swell gels. Nature 417, 388-391 (2002).

38. Pan, L. et al. Hierarchical nanostructured conducting polymer hydrogel with high electrochemical activity. Proc. Natl Acad. Sci. USA 109, 9287-9292 (2012).

39. Li, D. \& Kaner, R. B. Processable stabilizer-free polyaniline nanofiber aqueous colloids. Chem. Commun. 41, 3286-3288 (2005).

40. Wu, Y., Rahm, E. \& Holze, R. Carbon anode materials for lithium ion batteries. J. Power Sources 114, 228-236 (2003).

41. Wu, H. et al. Stable cycling of double-walled silicon nanotube battery anodes through solid-electrolyte interphase control. Nat. Nanotech. 7, 309-314 (2012).

\section{Acknowledgements}

We thank Dr Jeffrey B. Tok for helpful discussions. Y.C. and Z.B. acknowledge the funding support from the Precourt Institute for Energy at Stanford University, and from the Department of Energy, through the SLAC National Accelerator Laboratory LDRD project, under contract DE-AC02-76SF00515. L.P. thank the funding support from Natural Science Foundation of China (61076017, 60928009). M.T.M. acknowledges support from the NSF graduate fellowship and the Stanford Graduate Fellowship. This work was partially supported by the Assistant Secretary for Energy Efficiency and Renewable Energy, Office of Vehicle Technologies of the U.S. Department of Energy (contract no. DE-AC02-05CH11231) and the Batteries for Advanced Transportation Technologies (BATT) Program (subcontract no. 6951379).

\section{Author contributions}

H.W., G.Y., Z.B. and Y.C. conceived of the idea. H.W. and G.Y. performed the materials fabrication and electrochemical tests. L.P. provides polymer hydrogel materials. M.M. performed TEM characterizations. H.W., G.Y., Z.B. and Y.C. co-wrote the paper. All authors discussed the results and commented or revised the manuscript.

\section{Additional information}

Supplementary Information accompanies this paper at http://www.nature.com/ naturecommunications

Competing financial interests: The authors declare no competing financial interests.

Reprints and permission information is available online at http://npg.nature.com/ reprintsandpermissions/

How to cite this article: $\mathrm{Wu}, \mathrm{H}$. et al. Stable Li-ion battery anodes by in-situ polymerization of conducting hydrogel to conformally coat silicon nanoparticles. Nat. Commun. 4:1943 doi: 10.1038/ncomms2941 (2013). 